\section{Tratamiento antibiótico empírico de elección en pacientes con urosepsis secundaria a litiasis ureteral: reporte de sensibilidad local}

\author{
MANUEL CATALÁN ${ }^{1}$, INÉS CERÓN² ${ }^{2}$, GASTÓN ASTROZA ${ }^{1}$ \\ Antimicrobial susceptibility of bacteria
causing urosepsis
}

Background: Early inappropriate antibiotic therapy for the management of urosepsis is associated with higher mortality. Therefore, to establish an adequate empirical therapy is crucial. Aim: To determine an optimal antibiotic treatment, adjusted according local bacterial resistance in patients diagnosed with urosepsis secondary to ureteral lithiasis. Material and Methods: Urine cultures and blood cultures from a prospective cohort of patients with ureteral lithiasis admitted for urosepsis in our center between November 2013 and May 2016, were reviewed. Patients who presented two or more criteria of systemic inflammatory response syndrome (SIRS) and positive blood or urine cultures were admitted. Antimicrobial sensitivity patters derived from cultures were analyzed to describe bacterial susceptibility to commonly used antibiotics. Results: Data from 31 patients were analyzed. Seventeen blood cultures (55\%) and 29 urine cultures (94\%) were positive. The most commonly isolated pathogens were Escherichia coli in $65 \%$ of the cultures, followed by Klebsiella pneumoniae, Proteus mirabilis and Enterococcus faecalis. After performing an analysis of sensitivity and resistance for all bacteria in both blood and urine cultures, amikacin showed the highest sensitivity (100\%), followed by $2^{\text {nd }}$ and $3^{\text {rd }}$ generation cephalosporins. Conclusions: Amikacin demonstrated higher antibiotic sensitivity in comparison to other antibiotics for empirical management of patients with urosepsis secondary to ureteral lithiasis.

(Rev Med Chile 2017; 145: 755-759)

Key words: Anti-Bacterial Agents; Blood Culture; Lithiasis; Sepsis; Ureter; Urolithiasis.

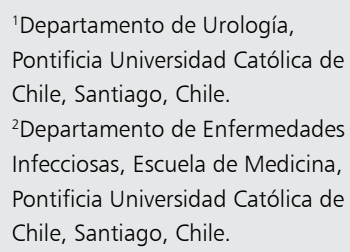

Recibido el 31 de enero de 2017. aceptado el 27 de junio de 2017.

No hubo financiamiento por parte de ninguna institución. Los autores no declaran conflictos de intereses.

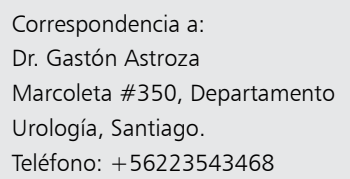

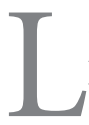

a sepsis de foco urinario o urosepsis es una patología sistémica que puede resultar como complicación de una ureterolitiasis, siendo esta su etiología más frecuente. En adultos, comprende hasta $25 \%$ de los casos de sepsis y las tasas de mortalidad varían entre $20-40 \% 1$.

Entre los factores de riesgo para su presentación encontramos: malformaciones genitourinarias, inmunodepresión (trasplante), edad avanzada, diabetes mellitus, entre otros ${ }^{2}$. Debido a su gran impacto en morbilidad y mortalidad, requiere un manejo precoz intensivo, tanto de soporte, como tratamiento antibiótico empírico. En los casos en los que se presenta asociado a obstrucción de vía urinaria, se debe realizar en forma asociada drenaje precoz de la vía urinaria ${ }^{1}$.

Algunos estudios han demostrado que una antibioterapia precoz inapropiada se puede asociar 
a mayores tasas de mortalidad ${ }^{3}$. Esto, sumado a la creciente resistencia antibiótica, hace imprescindible determinar el antibiótico empírico más adecuado a utilizar, ajustado a la microbiología local ${ }^{4}$.

Nuestro objetivo es determinar el tratamiento antibiótico empírico óptimo en la población de pacientes que se presentan con diagnóstico de sepsis de foco urinario secundaria a ureterolitiasis, ajustado por la resistencia bacteriana local.

\section{Materiales y Métodos}

Previa aprobación por el comité de ética de investigación, se realiza una cohorte prospectiva de pacientes portadores de ureterolitiasis ingresados con diagnóstico de urosepsis en el Departamento de Urología del Hospital Clínico de la Pontificia Universidad Católica de Chile, entre noviembre de 2013 y mayo de 2016. Los criterios de inclusión fueron: diagnóstico de litiasis a nivel ureteral demostrada mediante tomografía axial computada no contrastada (NC-CT), dos o más criterios de síndrome de respuesta inflamatoria sistémica (SIRS) y al menos un cultivo positivo, ya sea hemocultivo (HC) y/o urocultivo (UC), obtenidos antes del inicio del tratamiento antibiótico. Tanto los UC como los HC fueron procesados en el Laboratorio de Microbiología de nuestra institución. Los criterios de exclusión fueron: pacientes menores de 18 o mayores de 75 años, cálculos renales no obstructivos asociados a urosepsis y cuadros febriles con cultivos negativos.

De esta cohorte de pacientes, se realiza estadística descriptiva de características de la población estudiada, de los cálculos y análisis de los cultivos (UC y HC) con su respectivo antibiograma para caracterizar la sensibilidad y resistencia bacteriana a antibióticos (ATB) de uso habitual para cada cepa aislada. Finalmente, se propone el antibiótico de elección frente al planteamiento diagnóstico determinado por la menor tasa de resistencia en el total del grupo.

\section{Resultados}

Durante el período de estudio, de un total de 47 pacientes ingresados con diagnóstico de urosepsis, 31 pacientes cumplieron criterios de inclusión.
Las características generales de ellos se presentan en la Tabla 1.

En la totalidad de estos pacientes, la ureterolitiasis se demostró mediante tomografía axial computada no contrastada al momento de su ingreso hospitalario. Las características de la litiasis se detallan en Tabla 2.

En todos los pacientes se realizaron urocultivo y hemocultivo. Del total de hemocultivos, 17 resultaron positivos $(54,84 \%)$ y 14 fueron negativos $(45,16 \%)$. De los urocultivos, 29 resultaron positivos $(93,54 \%)$ y 2 negativos $(6,45 \%)$. Dentro de ellos, E. coli es la bacteria que se presentó de forma más frecuente en 20 casos $(64,6 \%)$. El resto de los microorganismos encontrados se detallan en la Tabla 3.

Un total de 15 pacientes $(48,3 \%)$ tuvieron ambos cultivos, HC y UC, positivos al mismo microorganismo, mientras que $11(37,9 \%)$ pa-

Tabla 1. Características de los pacientes

\begin{tabular}{|c|c|c|}
\hline Total Pacientes $=31$ & Masculino & Femenino \\
\hline Sexo & $\begin{array}{l}4 \text { pacientes } \\
(12,9 \%)\end{array}$ & $\begin{array}{l}27 \text { pacientes } \\
(87,1 \%)\end{array}$ \\
\hline Edad media & \multicolumn{2}{|c|}{ 43,5 años [18-75] } \\
\hline Creatinina al ingreso & \multicolumn{2}{|c|}{$1,4$ mg/dL (SD: 0,7$)$} \\
\hline APACHE II al ingreso & \multicolumn{2}{|l|}{8,3 (SD: 7,6) } \\
\hline Criterios SIRS & $\begin{array}{l}2(25,81 \%) \\
3(48,39 \%) \\
4(25,81 \%)\end{array}$ & \\
\hline $\begin{array}{l}\text { Tiempo medio total de } \\
\text { hospitalización }\end{array}$ & \multicolumn{2}{|c|}{7 días (SD: 3.8) } \\
\hline $\begin{array}{l}\text { Tiempo total de } \\
\text { hospitalización en UPC }\end{array}$ & \multicolumn{2}{|c|}{$\begin{array}{l}3 \text { días (SD: } 3,2) \\
11 \text { pacientes }(35,4 \%)\end{array}$} \\
\hline
\end{tabular}

Tabla 2. Características de la litiasis

\begin{tabular}{|c|c|c|c|}
\hline & $\begin{array}{l}\text { Uréter } \\
\text { proximal }\end{array}$ & $\begin{array}{l}\text { Uréter } \\
\text { medio }\end{array}$ & $\begin{array}{l}\text { Uréter } \\
\text { distal }\end{array}$ \\
\hline \multirow[t]{2}{*}{ Localización } & $\begin{array}{l}11 \text { cálculos } \\
(35,6 \%)\end{array}$ & $\begin{array}{c}5 \text { cálculos } \\
(16,1 \%)\end{array}$ & $\begin{array}{c}15 \text { cálculos } \\
(48,3 \%)\end{array}$ \\
\hline & \multicolumn{2}{|c|}{ Derecha } & Izquierda \\
\hline Lateralidad & \multicolumn{2}{|c|}{8 cálculos (25,8\%) } & $\begin{array}{l}23 \text { cálculos } \\
(74,2 \%)\end{array}$ \\
\hline Tamaño medio & \multicolumn{3}{|c|}{$5,95$ mm (SD: 3,02$)$} \\
\hline
\end{tabular}


cientes con UC positivo tuvieron $\mathrm{HC}$ negativo y $1(5,8 \%)$ paciente con $\mathrm{HC}$ positivo tuvo UC negativo. Ningún paciente presentó $\mathrm{HC}$ y UC con microorganismos distintos.

Las bacterias analizadas corresponden a bacilos gram negativos, a excepción de E. faecalis, siendo sensibles en $100 \%$ a amikacina, el único ATB analizado que muestra sensibilidad máxima en todas las cepas. Por otro lado, ampicilina demostró el perfil de sensibilidad más bajo, siendo $100 \%$ de las bacterias aisladas en algunos cultivos, resistentes. Los resultados se detallan en las Tablas 4 y 5.
Tabla 3. Bacterias identificadas en hemocultivos (HC) y urocultivos (UC)

\begin{tabular}{|lcc|}
\hline $\begin{array}{l}\text { Bacteria } \\
\text { identificada }\end{array}$ & $\begin{array}{c}\text { Hemocultivo } \\
(\mathbf{n}=\mathbf{3 1})\end{array}$ & $\begin{array}{c}\text { Urocultivo } \\
\text { (n = 31) }\end{array}$ \\
\hline Escherichia coli ${ }^{1}$ & $32,26 \%$ & $64,6 \%$ \\
\hline Klebsiella pneumoniae & $9,68 \%$ & $16,12 \%$ \\
\hline Proteus mirabilis & $12,9 \%$ & $6,45 \%$ \\
\hline Enterococus faecalis & 0 & $6,45 \%$ \\
\hline Ninguna & $45,16 \%$ & $6,45 \%$ \\
\hline
\end{tabular}

${ }^{1}$ De las cuales, $3,23 \%$ y $6,45 \%$ corresponden a cepas productoras de beta lactamasa de expectro extendido (BLEE), respectivamente para $\mathrm{HC}$ y $\cup C$.

Tabla 4. Bacterias identificadas en hemocultivos (HC) y su sensibilidad a antibióticos

\begin{tabular}{|c|c|c|c|c|}
\hline Hemocultivos $(n=31)$ & $\begin{array}{c}\text { Escherichia } \\
\text { coli }\end{array}$ & $\begin{array}{l}\text { E. Coli } \\
\text { BLEE }\end{array}$ & $\begin{array}{c}\text { Klebsiella } \\
\text { pneumoniae }\end{array}$ & $\begin{array}{l}\text { Proteus } \\
\text { mirabilis }\end{array}$ \\
\hline Amikacina & $100 \%$ & $100 \%$ & $100 \%$ & $100 \%$ \\
\hline Ampicilina & $33,3 \%$ & 0 & 0 & 0 \\
\hline Ampicilina/sulbactam & & 0 & $33,3 \%$ & $75 \%$ \\
\hline Cefazolina & $88,8 \%$ & 0 & $66,6 \%$ & 0 \\
\hline Cefotaxima & $100 \%$ & 0 & $66,6 \%$ & $100 \%$ \\
\hline Cefuroxima & $100 \%$ & 0 & $66,6 \%$ & $100 \%$ \\
\hline Ciprofloxacino & $88,8 \%$ & 0 & $66,6 \%$ & $100 \%$ \\
\hline Cloranfenicol & $100 \%$ & 0 & $33,3 \%$ & $75 \%$ \\
\hline Cotrimoxazol & $88,8 \%$ & 0 & $66,6 \%$ & $50 \%$ \\
\hline Gentamicina & $100 \%$ & 0 & $66,6 \%$ & $50 \%$ \\
\hline Carbapenémicos (imi-, mero-, ertapenem) & & $100 \%$ & & \\
\hline Piperacilina/tazobactam & & $100 \%$ & & \\
\hline
\end{tabular}

Tabla 5. Bacterias identificadas en uroocultivos (HC) y su sensibilidad a antibióticos

\begin{tabular}{|c|c|c|c|c|c|}
\hline Urocultivos $(n=31)$ & $\begin{array}{c}\text { Escherichia } \\
\text { coli }\end{array}$ & $\begin{array}{c}\text { E. Coli } \\
\text { BLEE }\end{array}$ & $\begin{array}{c}\text { Klebsiella } \\
\text { pneumoniae }\end{array}$ & $\begin{array}{l}\text { Proteus } \\
\text { mirabilis }\end{array}$ & $\begin{array}{c}\text { Enterococcus } \\
\text { faecalis }\end{array}$ \\
\hline Amikacina & $100 \%$ & $100 \%$ & $100 \%$ & $100 \%$ & \\
\hline Ampicilina & $33,3 \%$ & 0 & 0 & $50 \%$ & $100 \%$ \\
\hline Ampicilina/sulbactam & & 0 & $60 \%$ & $50 \%$ & \\
\hline Cefazolina & $72,2 \%$ & 0 & $60 \%$ & $50 \%$ & \\
\hline Cefotaxima & $94,4 \%$ & 0 & $80 \%$ & $100 \%$ & \\
\hline Cefuroxima & $94,4 \%$ & 0 & $80 \%$ & $100 \%$ & \\
\hline Ciprofloxacino & $77,7 \%$ & 0 & $80 \%$ & $100 \%$ & $50 \%$ \\
\hline Cotrimoxazol & $77,7 \%$ & $100 \%$ & $80 \%$ & 0 & \\
\hline Gentamicina & $100 \%$ & $50 \%$ & $80 \%$ & $50 \%$ & \\
\hline Nitrofurantoína & $94,4 \%$ & $50 \%$ & 0 & 0 & $100 \%$ \\
\hline $\begin{array}{l}\text { Carbapenémicos } \\
\text { (imi-, mero-, ertapenem) }\end{array}$ & & $100 \%$ & & & \\
\hline Piperacilina/tazobactam & & $100 \%$ & & & \\
\hline Vancomicina & & & & & $100 \%$ \\
\hline
\end{tabular}




\section{Discusión}

Si bien la literatura internacional sugiere opciones de terapia empírica antibiótica en situaciones como la analizada, es necesario que esta terapia sea ajustada a la microbiota local para un manejo más adecuado ${ }^{1}$. En Chile, existe literatura para el manejo de sepsis y shock séptico, que surge de una adaptación de guías internacionales, donde se sugiere manejo antibiótico empírico de amplio espectro de acuerdo a las características del paciente y los posibles patógenos ${ }^{5}$. Sin embargo, no existen estudios nacionales previos orientados específicamente a detallar el tratamiento empírico en urosepsis secundaria a ureterolitiasis.

En nuestro estudio, los antibióticos utilizados para llevar a cabo los antibiogramas de cada cultivo corresponden a antimicrobianos que se encuentran distribuidos ampliamente en la mayoría de los servicios hospitalarios a lo largo de nuestro país, lo que resulta especialmente importante cuando pensamos en su potencial validez externa.

El patógeno encontrado con más frecuencia fue E. coli, en $64,6 \%$ de los cultivos, como se ha descrito previamente en la literatura ${ }^{1,2,6}$. En segundo lugar, encontramos K. pneumoniae, seguido de $P$. mirabilis. La presencia de cepas resistentes de $E$. coli se ha asociado a: uso previo de antibióticos, uso de catéter urinario, origen intrahospitalario de infección, entre otros ${ }^{7}$. Esta situación es de considerar, puesto que cada vez es más frecuente en nuestro país, lo que hace perentorio el inicio de antibioticoterapia adecuada.

Evaluando globalmente la sensibilidad antibiótica, dentro del grupo de los aminoglicósidos, $100 \%$ de las bacterias aisladas en los cultivos fueron sensibles a amikacina; en cambio, en algunas cepas se observó hasta $50 \%$ de resistencia a gentamicina. Por otra parte, estudios han demostrado que la gentamicina es más nefrotóxica, al compararla con amikacina, y además esta toxicidad sería de presentación precoz ${ }^{8}$.

Basado en los hallazgos anteriores, planteamos que la amikacina es un buen antibiótico empírico para utilizar en sepsis de foco urinario, teniendo siempre en cuenta sus efectos adversos, tales como oto- y nefrotoxicidad ${ }^{9}$. Es por ello que resulta importante la evaluación inicial de antecedentes y de creatinina del paciente, para evaluar su función renal antes de iniciar el tratamiento y evitar que los daños superen a los beneficios.
Estos efectos adversos si bien se presentan hasta en $25 \%$ de los pacientes sometidos a tratamiento, son típicamente de aparición más tardía para amikacina; por ende, permitiría su uso empírico a la espera del resultado del antibiograma que pudiera permitir ajustar el tratamiento en el caso de pacientes con factores de riesgo de desarrollar toxicidad ${ }^{10,11}$.

A pesar del número reducido de casos, la distribución de bacterias en los cultivos fue similar a la reportada anteriormente en la literatura actual. La forma de obtención del urocultivo no fue especificada dentro del análisis de datos, además de las limitaciones propias de los estudios de laboratorio, sensibilidad y especificidad, tanto de urocultivo, como de hemocultivo. Si bien son los métodos utilizados para identificar los patógenos, su rendimiento no es máximo y las muestras podrían contaminarse $^{12}$.

Cabe mencionar además otros factores que pudieran influir en la selección del antibiótico, como: comorbilidades, antecedentes de hospitalización previa, colonización y/o infección previa por cepas resistentes.

\section{Conclusión}

La amikacina demostró un perfil de sensibilidad superior en comparación a los otros antibióticos, siendo $100 \%$ de las bacterias aisladas sensibles, para manejo empírico de pacientes con urosepsis secundaria a ureterolitiasis. Planteamos el uso de este antibiótico como terapia empírica óptima en este grupo de pacientes.

\section{Referencias}

1. Wagenlehner FM, Lichtenstern C, Rolfes C, Mayer K, Uhle F, Weidner W, et al. Diagnosis and management for urosepsis. Int J Urol 2013; 20 (10): 963-70.

2. Kalra OP, Raizada A. Approach to a patient with urosepsis. J Glob Infect Dis 2009; 1 (1): 57-63.

3. Elhanan G, Sarhat M, Raz R. Empiric antibiotic treatment and the misuse of culture results and antibiotic sensitivities in patients with community-acquired bacteraemia due to urinary tract infection. J Infect 1997; 35 : 283-8.

4. García CP. Resistencia bacteriana en Chile. Rev Chilena Infectol 2003; 20 (Supl. 1): 11-23. 
5. Dellinger R, Levy M, Rhodes A, Annane D, Gerlach $\mathrm{H}$, Opal S, et al. Campaña para sobrevivir a la sepsis: recomendaciones internacionales para el tratamiento de sepsis grave y shock séptico, 2012. Crit Care Med 2013; 41.

6. Thomas M, Hooton MD. Acute complicated cystitis and pyelonephritis. Jul 2016. In: UpToDate.

7. Al Yousef SA, Younis S, Farrag E, Moussa HSh, Bayoumi FS, Ali AM. Clinical and Laboratory Profile of Urinary Tract Infections Associated with Extended Spectrum $\beta$-Lactamase Producing Escherichia coli and Klebsiella pneumoniae. Ann Clin Lab Sci 2016; 46 (4): 393-400.

8. Sweileh WM. A prospective comparative study of gentamicin- and amikacin-induced nephrotoxicity in patients with normal baseline renal function. Fundamental \& Clinical Pharmacology 2009; 23: 515-20.

9. Wargo KA, Edwards JD. Aminoglycoside-induced nephrotoxicity. J Pharm Pract 2014; 27: 573-7.

10. López-Novoa JM, Quiros Y, Vicente L, Morales AI, López-Hernández FJ. New insights into the mechanism of aminoglycoside nephrotoxicity: an integrative point of view. Kidney Int 2011; 79 (1): 33-45.

11. Sandhu JS, Sehgal A, Gupta O, Singh A. Aminoglycoside nephrotoxicity revisited. J Indian. Acad Clin Med 2007; 8 (4): 331-3.

12. Hall KK, Lyman JA. Updated Review of Blood Culture Contamination. Clin Microbiol Rev 2006; 19 (4): 788 802. 